\title{
IMPROVING 3D MESH QUALITY USING MULTI-DIRECTIONAL UAV IMAGES
}

\author{
Jihun Kang, Sewon Lee, Sunghyun Yeon, Seongjin Park \\ Spatial Information Research Inst., Korea Land and Geospatial Informatix Corp., Republic of Korea - (kangdaejang, leesewon, \\ yeon)@1x.or.kr \\ Sistech Corp., Seoul, Republic of Korea - signs00@daum.net
}

KEY WORDS: UAV, Oblique image, Nadir image, Point cloud, 3D, Mesh

\begin{abstract}
:
With the increasing use of 3D spatial information, updated 3D spatial information is required and unmanned aerial vehicles (UAVs) are being used to accomplish this task. In general, 3D spatial information is constructed by generating meshes through point clouds extracted from many images. However, generating a mesh that is comparable to a real building is difficult because UAV images use relatively cheap sensors compared to aerial images. In this study, Nadir images with various overlapping degrees were obtained and point clouds generated to compare the reproducibility of actual buildings. This led to the confirmation that the use of Nadir images alone does not fully complement the occlusion even when the images are taken with high overlapping degrees. To complement such limitations of unidirectional images, oblique images were used in addition to create point clouds, and the occlusion was found to have been complemented. Furthermore, through its conversion, high quality 3D spatial information data could be obtained.
\end{abstract}

\section{INTRODUCTION}

Construction of 3D spatial information using aerial images can generate high quality data, but it is quite expensive and timeconsuming. For certain city areas with increased demand for 3D spatial information, changes in buildings occur frequently, but 3D spatial information is not regularly updated due to cost restrictions. Recently, 3D spatial information construction using unmanned aerial vehicle (UAV) images has been attempted as a result of UAV technology development and governmental deregulation. In particular, rotor wing UAVs with easy flight and portability are emerging as an alternative method for updating $3 \mathrm{D}$ objects in small areas.

Construction of 3D spatial information using UAVs is generally performed by first generating point clouds with many images taken by the UAVs, converting the point clouds to mesh data, and subsequently modeling and texturing to create objects with realistic forms via after-treatment. Similar to existing aerial photographic methods, the UAV method constructs point clouds by overlapping images taken from the vertical direction (Nadir) and via geometric interpretation (Tomi and Eija, 2012; Rhee and Kim, 2016; Song and Um, 2017; Rhee et al., 2018). However, acquiring accurate point clouds from UAVs is difficult because they are not equipped with the expensive sensors used for aerial images. To solve this problem, many studies have attempted to enhance and evaluate the accuracy of UAV obtained point clouds (Steve and Arko, 2012; Choi et al., 2016; Lee and Lee, 2017).

For or that, point clouds with high locational accuracy do not guarantee good reproduction of building shapes. Point clouds should be evenly distributed on the outer area of buildings without empty areas to correctly represent the building shapes. However, for high-rise buildings point clouds are usually not correctly formed in the lower parts or occlusion areas, and problems such as close holes can occur, which appear as holes in the building. These are the main reasons for the low mesh quality of buildings, which can result in rendering of a different shape compared to that of the real building. Increasing the degree of overlap is a potential solution to this problem, but it has its own limitations.
In this study, UAV images were obtained for a building with different overlap degrees and point clouds were subsequently created to determine the number of close holes in the point clouds as a function of overlapping. To minimize the occurrence of close holes in the point clouds, oblique images were obtained from circular flight and used to construct the point cloud.

\section{TEST METHOD}

\begin{tabular}{|c|c|}
\hline Component & Detail \\
\hline Material & Carbon, Aluminium \\
Size & $\begin{array}{c}4.2 \mathrm{~kg} \\
\text { Weight } \\
\text { (Includes battery and A6000 } \\
\text { camera) }\end{array}$ \\
$\begin{array}{c}\text { Takeoff weight } \\
\text { Mission Equipment Weight } \\
\text { Battery }\end{array}$ & $\begin{array}{c}600 \mathrm{mg} \\
\text { Flight time } \\
\text { Velocity }\end{array}$ \\
& Max $300 \mathrm{~mm}$ \\
& \\
\hline
\end{tabular}

Table 1. The specifications of the test vehicle (Kmapper)

The test target was an 8-floor building of approximately $40 \mathrm{~m}$ height, located in Ilsan, Gyeonggi province, Republic of Korea. The test area was selected because the photographing of flights was easily permitted and it was composed mostly of rice fields 
and orchards with a small number of people in the area around the building to allow a safe flight test. Moreover, the building was characterized by having more than half its materials based on glass as is the case with urban buildings. Its close proximity to a 3 -floor building made it appropriate for the test.

The UAV used in the test was Kmapper of Sistech, a frame with rotary wings for mapping. Kmapper is able to photograph approximately $1 \mathrm{~km}^{2}$ per flight, while allowing the installation of various sensors such as a camera or a multispectral sensor via a mount for mission devices. The specifications and images of Kmapper are presented in Table 1. In this study, the camera attached to Kmapper was A6000 of Sony.

To examine how the changes in UAV images with respect to the overlapping degrees and directions in photographing affect the point clouds, two separate tests were carried out. The test with different overlapping degrees were performed using only with Nadir photographs, which are taken at $90^{\circ}$ from UAV toward the ground, and the obtained images exhibited $60 \%, 70 \%$, and $85 \%$, respectively, for flight directions and side overlaps. In addition, oblique images were taken to obtain a sideview of the building in addition to the Nadir images, to test the effect of photographing direction. Oblique photographing was performed by circular flight around the tested building, with a $30^{\circ}$ incline from the UAV to the ground. Four flights in total were performed to carry out the two tests, and the flight paths for each test are as shown in Figure 1.

\section{CREATION AND COMPARISON OF POINT CLOUDS}

Point clouds were extracted from the images taken by the UAV, then visualized, and to compare the reproduction with the real building, the Photoscan software of Agisoft was used. First, the result of the flight where photographing directions were fixed by Nadir and overlapping degrees were modified, is given in Table 2. To remove any variables other than the overlapping degrees, images were taken while maintaining identical area and altitude.

\begin{tabular}{|c|ccc|}
\hline Category & $60 \%$ & $70 \%$ & $85 \%$ \\
\hline Flight area & \multicolumn{3}{|c|}{$300 \mathrm{~m} \times 300 \mathrm{~m}$} \\
Flight altitude & \multicolumn{3}{|c|}{$100 \mathrm{~m}$} \\
Flight duration & $8 \mathrm{~min} 30 \mathrm{sec}$ & $10 \mathrm{~min}$ & $18 \mathrm{~min} 30 \mathrm{sec}$ \\
Images taken & 76 images & 147 images & 332 images \\
\hline
\end{tabular}

Table 2. Nadir photographing records with different overlapping degrees

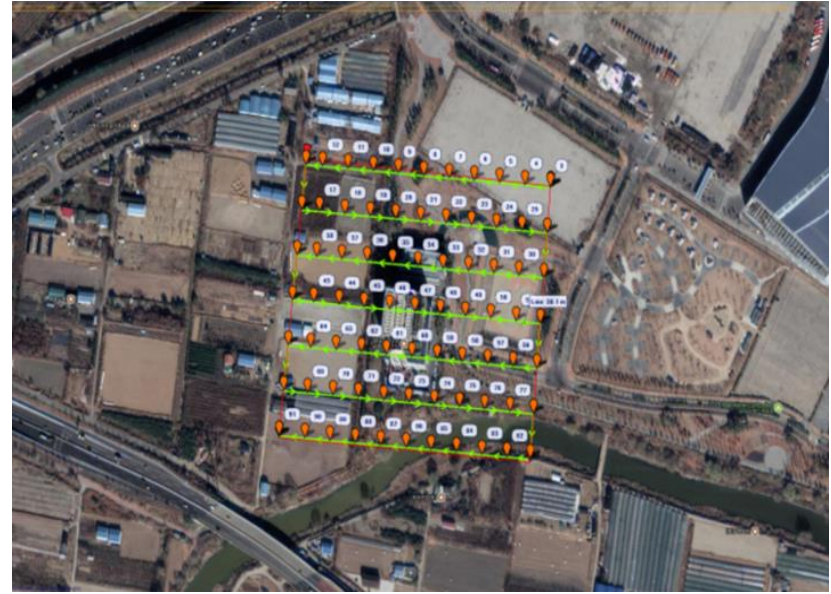

(a) Nadir $(60 \%)$

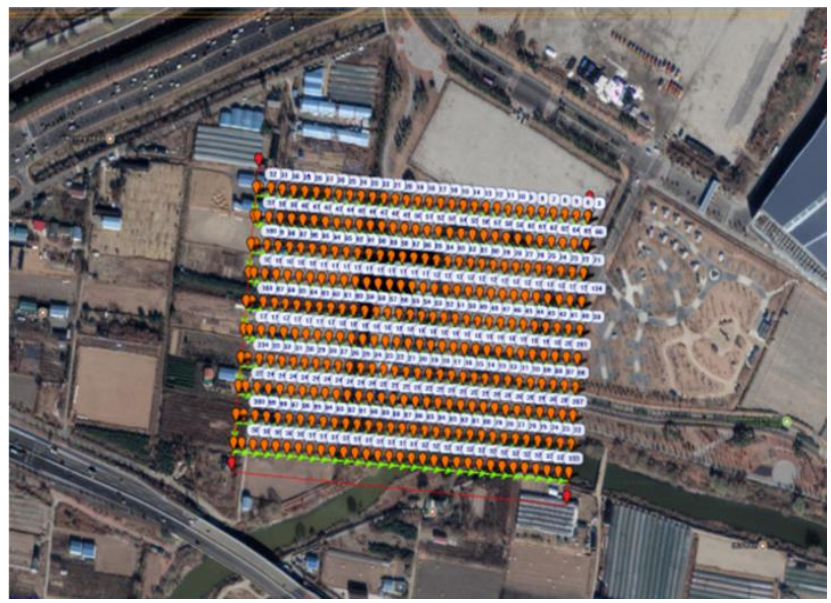

(c) Nadir (70\%)

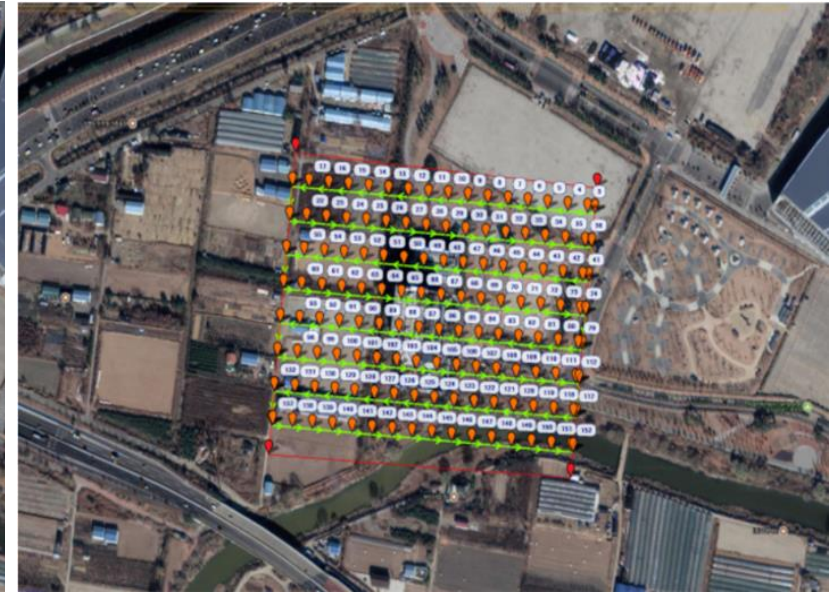

(b) Nadir (70\%)

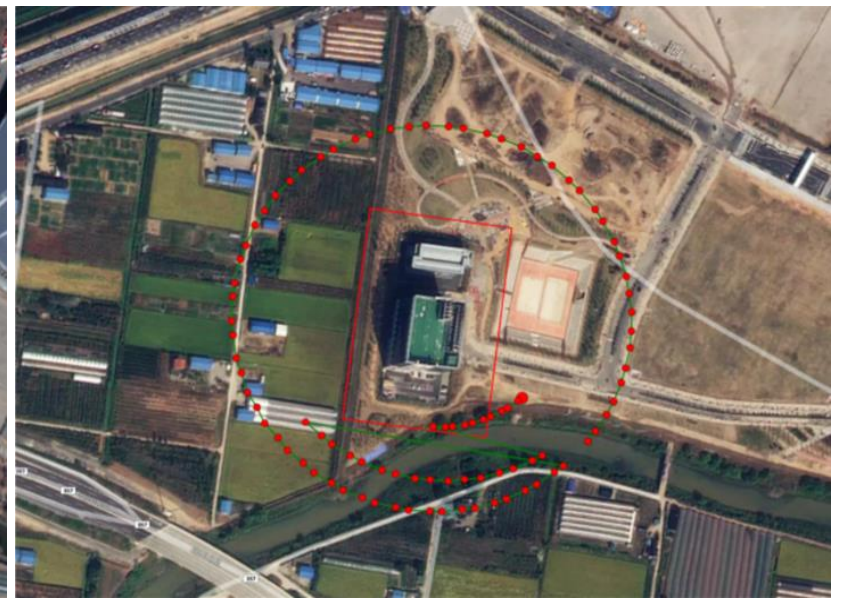

(d) Oblique

Figure 1. Test flight method and plan 


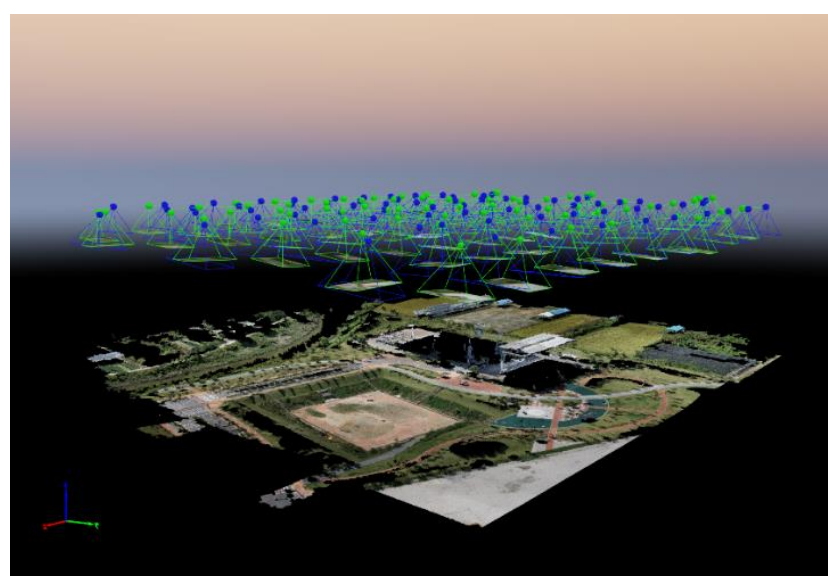

(a) Perspective view of $60 \%$ overlap

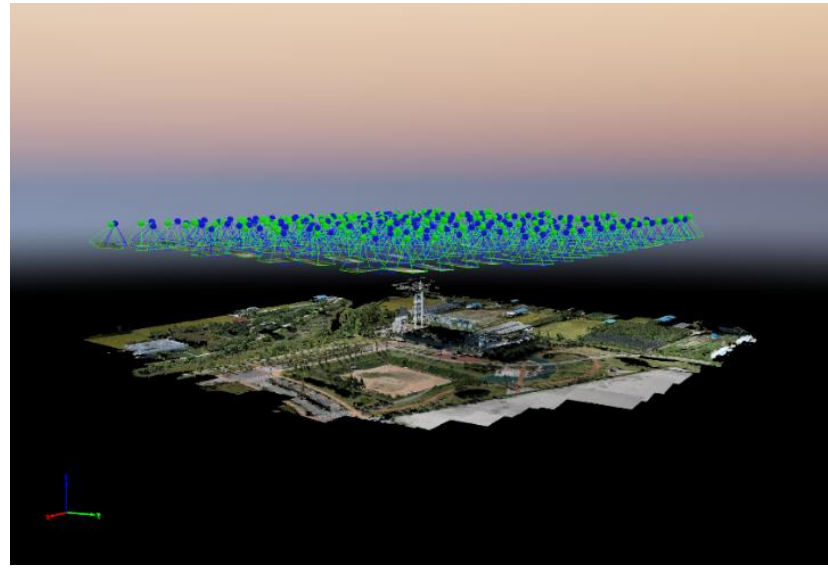

(c) Perspective view of $70 \%$ overlap

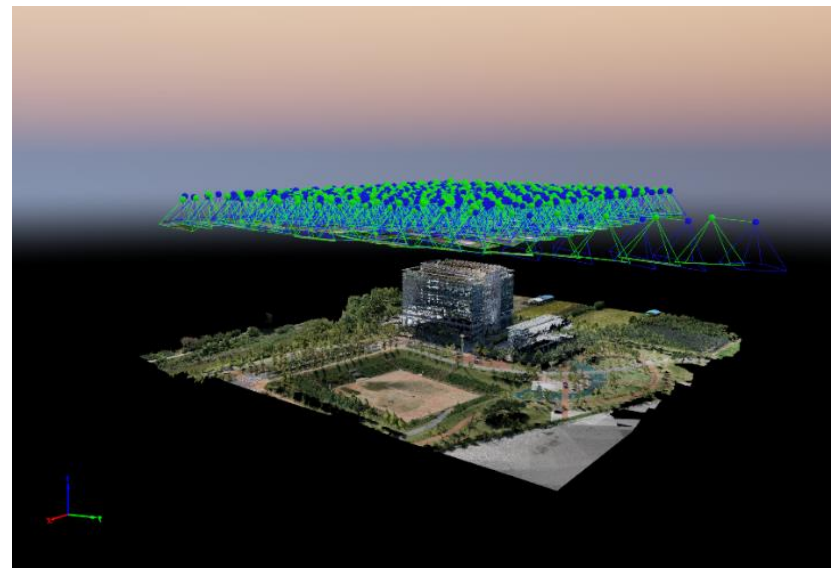

(e) Perspective view of $85 \%$ overlap

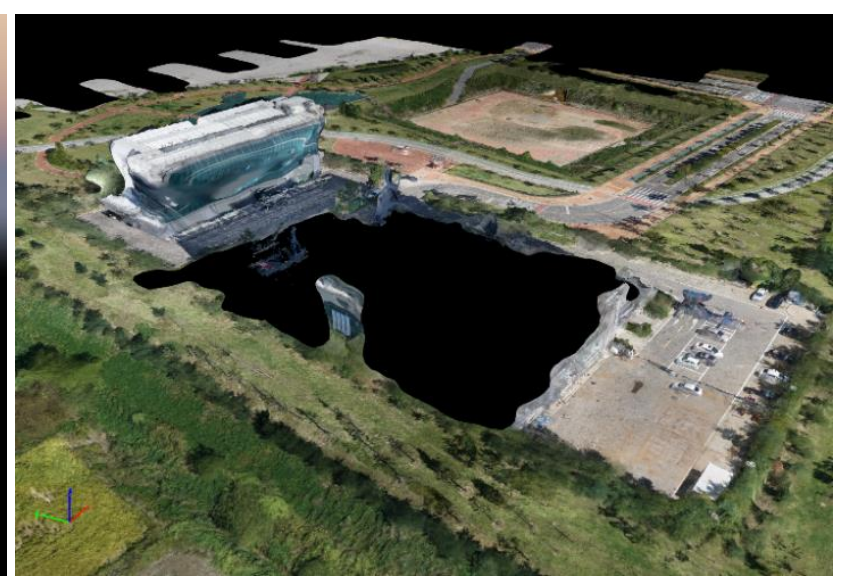

(b) Close view of $60 \%$ overlap

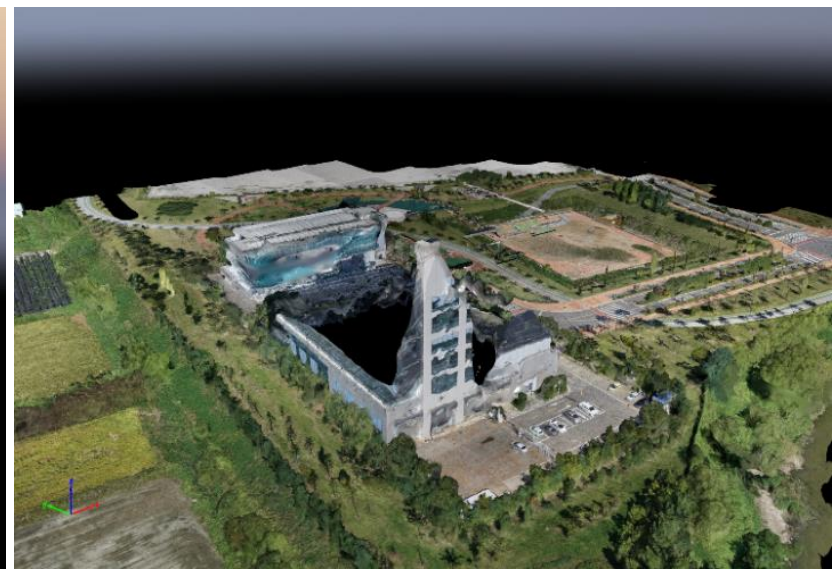

(d) Close view of $70 \%$ overlap

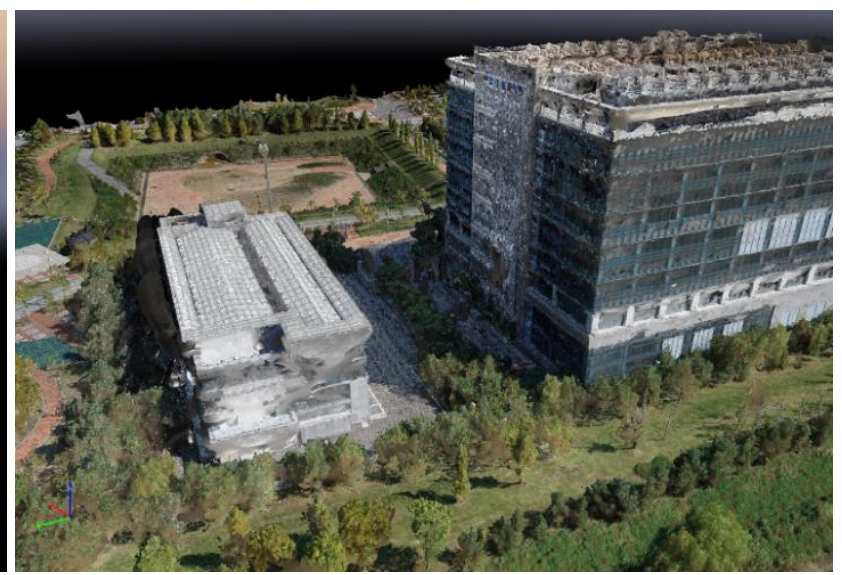

(f) Close view of $85 \%$ overlap

Figure 2 Photographing points and point clouds

The point cloud results with different overlapping degrees created from images taken at various photographing points are shown below (Figure 2). The point clouds generated from the UAV images with $60 \%$ overlap reproduced the surrounding landscape, but the buildings were poorly reproduced. The test building did not generate any point cloud at all, and only the roof side of the neighboring 3 -floor building led to the creation of point clouds.

The point clouds extracted from the images with $70 \%$ overlap resulted in the reproduction of numerous parts of the building compared to the case of $60 \%$, but still the overall building shape was not reproduced. The test building generated the point clouds only for a part of a single side wall, whereas the neighboring building reproduced the shape of the roof as well as the side of the building despite ambiguity.

The point clouds generated from the UAV images with $85 \%$ overlap reproduced the buildings and landscape realistically in a distance view. In addition to the neighboring building, point clouds were extracted from the roof and four side walls of the test building. However, the occlusion area of the test building and the neighboring building did not allow proper generation of point clouds (Figure 2(f)). 
Next, the result of photographing oblique images for extracting point clouds using multidirectional drone images, is as shown in Figure 3. A total of 400 images were taken, and combined with the previously obtained 40 Nadir images to generate the point clouds. Here, the Nadir images used were those extracted among the images taken with $70 \%$ overlapping degrees in the order of proximity between the photographing center and the building center. The reason why the images of $70 \%$ overlapping degrees were used was to confirm whether the use of oblique images led to the decrease in overlapping degrees when Nadir images were photographed.

Based on the result of generating point clouds, the previously occluded sides of the test building and neighboring building were reproduced similarly to the real buildings. In particular, the usual close-hole phenomenon in the lower part of the high-rise building and occlusion areas was hardly observed.

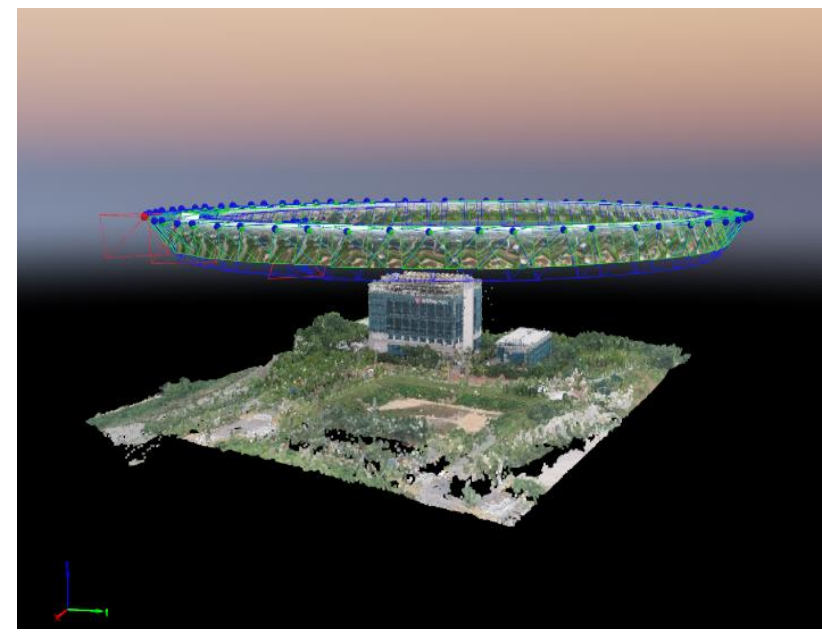

Figure 3 Oblique image photographing points and point cloud generation results.

The point clouds extracted using multidirectional drone images were converted to produce the building mesh. The modelling using the DSM texturing function of Photoscan software led to the creation of a clean model as shown in Figure 4. A complex structure of air conditioning units on the roof of the building prevented proper generation of mesh, posing a limitation to the creation of point clouds through image matching. Nevertheless, excluding the roof, production of a high quality $3 \mathrm{D}$ mesh was confirmed for the topography as well as the sides of the test building and the neighboring building.

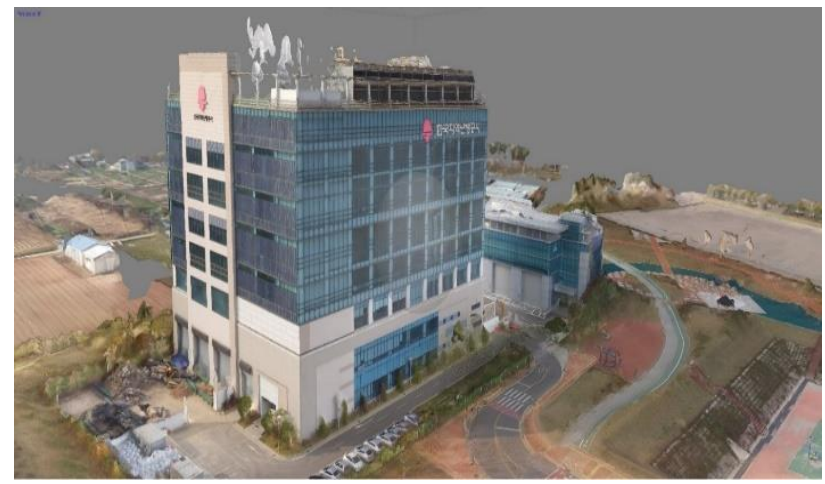

Figure 4 The 3D model generated by combining oblique and Nadir images.

\section{CONCLUSION}

The demand for 3D spatial information data is continuously rising in diverse fields including disaster management, virtual training, and game. What is required to meet such demand is not only the accuracy of data but also the realistic and rapid update of data. The technology that automatically generates 3D data through drone image matching is a prerequisite. To find a way to use drone images for generating high quality $3 \mathrm{D}$ data, this study examined the effect of overlapping degrees of Nadir images on 3D building reproduction, and tested how combining Nadir and Oblique images affected 3D building reproduction.

The result of the test where point clouds were extracted from Nadir images with varying overlapping degrees, showed that point clouds more similar to the real buildings were obtained with high overlapping degree. Almost no point clouds were generated for the building excluding the topography upon $60 \%$ overlapping degrees, while $85 \%$ overlapping degrees led to the generation of point clouds similar to the actual building in terms of the roof and the side walls. However, even upon photographing with $85 \%$ overlapping degrees, the maximum level used in this study, a limitation was found as point clouds were not properly generated in the occlusion area between the test building and the neighboring building.

In contrast, the result of extracting point clouds by combining oblique images and Nadir images of $70 \%$ overlapping degrees, showed a closer reproduction of the test building. Although the Nadir images of only $70 \%$ overlapping degrees were used, the point clouds for the occlusion area were properly generated, while they could not be so well using $85 \%$. This confirmed that the use of oblique images enabled an effective generation of point clouds with Nadir images of lower overlapping degrees. Also, when such point clouds created by multidirection images were applied to the generation of 3D mesh through the texturization and subsequent visualization, high reproducibility of the real building was confirmed.

The result of the test indicated that, to obtain 3D mesh data similar to the actual building, the use of multidirectional UAV images was essential. However, for an efficient construction of high quality $3 \mathrm{D}$ data, additional tests are required to determine the optimal photographing angles, directions, and overlapping degrees for obtaining oblique images. Further studies should also investigate the way to efficiently combine oblique images and Nadir images, whereby point clouds may be extracted even for a structure with high complexity.

\section{ACKNOWLEDGEMENTS}

This research was supported by a grant (19DRMSB147287-02) from development of customized realistic 3D geospatial information update and utilization technology based on consumer demand, funded by Ministry of Land, Infrastructure and Transport of Korean government.

\section{REFERENCES}

Choi, J.W., Choi, S.K., Yoo, Y.H., Jung, S.H., 2016. Quality Evaluation of Orthoimage and DSM Based on Fixed-Wing UAV Corresponding to Overlap and GCPs. Journal of the Korean Society for Geo-spatial Information Science, 24(3), 3-9.

Lee, K.R. and Lee, W.H., 2017. Comparison of Orthophoto and 3D Modeling using Vertical and High Oblique Images taken by 
UAV. The Korean Society for Geospatial Information System, 25(4), 35-45

Rhee, S. and Kim, T., 2016. Dense 3D Point Cloud Generation from UAV Images from Image Matching and Global Optimization. Proc. of 2016 International Archives of the Photogrammetry, Remote Sensing and Spatial Information Sciences, Prague, Czech Republic, Vol. XXIII, pp. 1005-1009.

Rhee, S., Hwang, Y., Kim, S., 2018. A Study on Point Cloud Generation Method from UAV Image Using Incremental Bundle Adjustment and Stereo Image Matching Technique. Korean Journal of Remote Sensing, 34(6), pp. 941-951.

Song, Y. and Um, D., 2017. Development of Classification Technique of Point Cloud Data Using Color Information of UAV Image, Journal of the Korean Society of Surveying, Geodesy, Photogrammetry, and Cartography, 35(4), 303-312.

Steve, H. and Arko, L., 2012. Assessing the Accuracy of Georeferenced Point Clouds Produced via Multi-View Stereopsis from Unmanned Aerial Vehicle (UAV) Imagery. Remote Sensing, 4(6), 1573-1599.

Tomi, R. and Eija, H., 2012. Point Cloud Generation from Aerial Image Data Acquired by a Quadrocopter Type Micro Unmanned Aerial Vehicle and a Digital Still Camera. Sensors, 12(1), 453380. 